(and the use of approved tools such as SPICT) and the need for increased education for GPs about all aspects of supportive end of life care and advanced care planning.

\section{THE COST OF A NIGHT NURSING SERVICE AT RENNIE GROVE HOSPICE CARE, AND THE TOTAL COMMUNITY COST OF CARE AT HOME COMPARED TO AN ADMISSION TO HOSPITAL}

S Varvel, SG Spiro, A Graham, J Sixsmith, A Ward. Rennie Grove Hospice Care, The University of Northampton, The University of Dundee

\subsection{6/bmjspcare-2018-ASPabstracts.66}

Background Rennie Grove (RG) runs a 24/7 Hospice at Home service. An independent study calculated the cost of a visit and the total community cost of home care, including all health care professional (HCP), carer, and family member visits.

Methods Over a period of 145 days, 550 calls and 335 visits made to/by the night team were recorded, averaging 3.79 per night. The salary cost per hour for each nurse, plus organisational add on costs, were calculated. To derive a total community cost, 35 families, able to consent, kept a diary for up to two-weeks, recording all HCP, carer and family support visits and duration of each visit. 17 diaries were returned. Descriptive analysis was used with the Statistical Package for the Social Sciences (SPSS v22). Costs were taken for HCPs and social care services from the PSSRU data 2015/2016.

Results Average length of the diaries was 10.4 days. For 3.79 visits per night the cost per visit was $£ 195$ (RG nurses travel in pairs). 17 patient diaries covered 177 days and showed a range of visits and complexity of care. RG staff provided $19 \%$ of the care at a cost of $£ 3,295$; district nurses $13 \%$, cost $£ 2,005$; formal carers $55 \%$ at a cost of $£ 1344$. 23 GPs visits comprised $4 \%$ of visits but $15 \%$ of total costs. MacMillan/Marie Curie nurses accounted for just 2\% of visits but $19 \%$ of cost as they stayed overnight. The cost of 177 days of care for 17 patients was $£ 11,814$; i.e. $£ 66.7$ per day as care was not needed every day of each diary period.

Conclusions The cost of home care seems acceptable, compared to the national average cost of a day in an inpatient specialist palliative care bed at $£ 397-£ 400$ (Data.Gov.UK, 2015).

\section{HOW SATISFIED ARE CARERS/FAMILIES WITH A NIGHT TEAM SERVICE AS PART OF 24/7 HOSPICE AT HOME CARE?}

S Varvel, A Ward, J Sixsmith, A Graham, SG Spiro. Rennie Grove Hospice Care, The University of Northampton, The University of Dundee

\subsection{6/bmjspcare-2018-ASPabstracts.67}

Background Rennie Grove (RG) runs a 24/7 service with a dedicated responsive night team. Through an independent study, carers' levels of service satisfaction with the night team were investigated.

Methods A questionnaire was sent to carers $(n=268)$ who had a relative die under the care of RG within the last 6 weeks to one year, 84 completed questionnaires were returned. Follow-up face-to-face semi-structured interviews were carried out with carers $(n=18)$. Quantitative data was analysed using SPSS v 22, while qualitative data was thematically analysed.

Results The carer questionnaire reported that 71\% thought the speed of the response by the night team was fast. Overall satisfaction with telephone call response was high with $82 \%$ very satisfied. Carers also agreed strongly that the telephone calls provided good quality of care (90\%), good follow-up care $(88 \%)$, that they were given sufficient time $(88 \%)$, and received useful advice (83\%). For a visit, 93\% of carers were highly satisfied and $7 \%$ satisfied. Carers strongly agreed that they were given sufficient time (90\%), and were confident in the care provided (90\%), care was useful (87\%) and followup was good (84\%), worries were reduced (81\%) and they had a say in decisions made (81\%). During the interviews, carers described the night service as: supportive, amazing, caring, reassuring, professional, kind and compassionate, excellent, loving, calm. Carers perceived the service to be holistic and rated the service highly for supporting them to ensure family members could be cared for at home, as preferred. For comparison, of those dying in hospital, only $46 \%$ were highly satisfied. Carer wellbeing scores were lower than the national average, but reported similar levels of anxiety.

Conclusions A night team service can provide excellent, reassuring and holistic care that meets the care needs and wishes of the patient and family.

\section{DOES A 24/7 HOSPICE AT HOME SERVICE PREVENT OR POSTPONE ACUTE HOSPITAL ADMISSIONS?}

SG Spiro, A Ward, A Graham, J Sixsmith. Rennie Grove Hospice Care, The University of Northampton, The University of Dundee

\subsection{6/bmjspcare-2018-ASPabstracts.68}

Background Most people wish to die at home but in England almost 50\% die in hospital, suggesting that more can be done to keep people at home. Some studies have shown this may be possible, especially with adequate support and good pain control.

Methods Rennie Grove Hospice Care carried out an independent study to identify the value of their night team in providing 24/7 care over a period of 145 nights. The study considered whether the provision of overnight care affected decisions to make hospital admissions. Data from a night nurse template recording all overnight visits, a review of 42 patient/carer records of those who had called the night team, a carer questionnaire $(n=87)$, carer interviews $(n=18)$ and staff interviews $(n=9)$, were analysed.

Results The night nurse template recorded 5 overnight admissions made during the study period, all for acute reasons and considered unavoidable. Review of 42 patient records showed 23 hospital/hospice admissions occurred, most during the daytime (18 to hospital, 5 to a hospice) of which only 3 were considered avoidable. The carer questionnaires showed 13 (16\%) of their cared for persons had been admitted to hospital in the last few weeks of life, with most reporting the admission was necessary. Staff interviews identified a pride in supporting patients to stay at home, while information from carers gave a clear indication that Rennie Grove nurses enabled their cared for individual to remain at home; that contact with the night team helped prevent or postpone an admission; and without Rennie Grove their alternative would have been 111/999, district nurse or out-of-hours GP. 
Conclusions There are occasions when hospital admission is appropriate. However, there was clear evidence showing support from the night team could prevent or postpone hospital admissions.

\section{THE VALUE OF A NIGHT SERVICE FOR HOSPICE AT HOME; REASONS FOR MAKING CONTACT AT NIGHT}

SG Spiro, A Ward, J Sixsmith, A Graham. Rennie Grove Hospice Care, The University of Northampton, The University of Dundee

\subsection{6/bmjspcare-2018-ASPabstracts.69}

Background People approaching the end of their lives have physical and psychological needs that should be met in a timely way, at any point during the night or day, and met in a way which addresses their needs and preferences. Crises at any time requires prompt, safe and effective urgent care (NICE 2011).

Methods Rennie Grove (RG) provides 24/7 care, with a night service available from $9.15 \mathrm{pm}$ to $7.15 \mathrm{am}$. RG conducted an independent study to identify the value of the service, including reasons people make contact at night and how many calls could be settled without a visit. Over the study period (145 nights) each call was charted with demographic details, reason for call and outcome.

Results 550 calls were received, resulting in 335 visits. On 7 nights there were no visits, the highest number in a night was 9, with an average 3.79 visits per night. Only those consenting to participate were analysed, reducing eligibility to 351 calls and 208 visits. Of these, reasons for a night visit included symptom control (64\%), carer support (19\%), death (13\%), death verified by night team (9\%), message (7.5\%), planned visit (7\%) and 'other' (4\%). Major symptoms included pain (42\%), anxiety (32\%), nausea (17\%) and a small number with chest problems, bowel issues, pyrexia, syringe pumps. In 143 cases a visit was not necessary due to telephone reassurance (55\%), another service being more suitable $(12 \%)$, the team were too busy $(8 \%)$, and $12 \%$ were handed to the next shift.

Conclusions The night team plays a vital role in $24 / 7$ care, with $40 \%$ of calls being managed without a visit. Visits were, in the main, for symptoms or problems concerning support. Death, for a Hospice at Home service also becomes a significant issue requiring a prompt and caring response.

\section{PERSONALISED CARE PLANNING TOOL FOR DYING PATIENTS; PEOPLES PERCEPTIONS AND IMPACT ON CARE}

Kate Tredgett, Karen Brown. Great Western Hospitals NHS Foundation Trust Swindon

\subsection{6/bmjspcare-2018-ASPabstracts.70}

Introduction In response to 'One chance to get it right' we developed a local tool to support the care of dying people in an acute hospital. It comprised a document with guidance and tear out elements for clinical staff and a patient/family information book. We report on the data acquired on the tool's impact on care and the perceptions of relatives and clinicians. Methods and results -Impact on care; This was evaluated through feedback from bereaved families using a validated questionnaire. Questionnaire responses were compared for the deaths supported and not supported by the tool. Overall score for quality of care from 123 completed questionnaires was $16 \%$ higher when care was supported by the tool $(72 \% \mathrm{v}$ $88 \%)$.

-Clinician Feedback; More than $75 \%$ of Trust staff have completed mandatory video based e.learning training in care of the dying and in using the tool. Feedback was collected from 60 medical and nursing staff of mixed grades following a learning event. $63 \%$ rated the tool 'very helpful' with the remainder rating it 'quite helpful'. Comments included that the tool provides 'written evidence of discussions' and 'staff are aware of the patient's wishes,' and highlighted practical issues including that it is necessarily extensive and takes a long time to complete'.

-Family Feedback; In addition to the completing the validated questionnaire, families were invited provide feedback on the tool itself. $96 \%$ of respondents rated it very or quite helpful with comments including 'discussed in detail' and 'I'm glad it was there'.

Discussion The findings suggest that the tool is helpful. Because deaths not supported by the tool may differ systematically from those supported by the tool, there may be bias which could dilute the tool's impact as evaluated by the questionnaire. Clinician and family feedback support its ongoing use and continued development.

\section{PRELIMINARY OUTCOME ANALYSIS OF INTEGRATED CARE FOR ADVANCED RESPIRATORY DISORDER (ICARE) - A MULTIDISCIPLINARY PALLIATIVE REHABILITATION PROGRAM FOR ADVANCED LUNG DISEASE IN A COMMUNITY HOSPITAL}

Nicholas Lee, Neo Han Yee. Lee Kong Chian School of Medicine Nanyang Technological University Singapore, Tan Tock Seng Hospital Singapore, Ren Ci Hospital Singapore

\subsection{6/bmispcare-2018-ASPabstracts.71}

Background Dyspnea is a prevalent and debilitating symptom, especially in patients with advanced pulmonary diseases. Dyspnea support services have been shown to improve functional outcomes and quality of life. This report presents a retrospective audit of a novel inpatient dyspnea support service for advanced respiratory diseases in Singapore.

Methods ICARE employs multidisciplinary dyspnea management, pulmonary rehabilitation, structured comorbidity screening using CO-morbidity Assessment Template (COAT), and dyspnea self-management interventions for patients discharged from Respiratory Medicine. The primary outcomes assessed were 6 min Walk Distance (6MWD) and Modified Barthel Index (MBI). Secondary outcomes include clinical issues identified by COAT that were treated, as well as 30 day readmissions.

Results ICARE had 39 unique admissions with 4 recurrent patients. The patients were mostly elderly Chinese males who were exceptionally frail with considerable co-morbidity. Their mean age was $73.2( \pm 9.5)$ years, $87.1 \%$ had COPD of which $81.8 \%$ was GOLD stage 3/4. Admission 6MWD was $130.4 \mathrm{~m}$ ( \pm 90.6$) .3 .3( \pm 1.3)$ of 5 basic ADLs were affected by dyspnea. They had mean 5.1 respiratory hospitalisations 1 year prior to ICARE admission with median hospital LOS 30.0 (IQR 20.0-60.0) days, 12.8\% had 10 hospitalisations.

The median program duration was 24.0 (IQR 12-35) days. 6MWD improved by median $30 \mathrm{~m}$ (IQR 20-60) $(\mathrm{p}=0.05)$. 